\title{
The effect of encoding variables on the free recall of words and action events
}

\author{
RONALD L. COHEN \\ Glendon College, York University, Toronto, Ontario, Canada
}

\begin{abstract}
On the basis of previously determined properties, it was hypothesized that, whereas the free recall of words is a strategic memory test, the free recall of action events in the form of tasks performed by the subject is nonstrategic. This hypothesis was tested in two experiments. In Experiment 1, some presented items were designated as being more important to remember than others. Although this encoding manipulation had a marked effect on word recall, its effect on task recall was minimal. In Experiment 2, the encoding impact of events was measured by having subjects rate, during presentation, how likely they were to recall each event on a subsequent test. The ratings showed good predictive power for the recall of words, but not of action events. These results were accepted as further support for a strategic/nonstrategic distinction between word and task recall.
\end{abstract}

In a recent article, Cohen (1981) described some of the properties of a heretofore neglected class of memory events, namely, action events that involve subject performance (subject-performed tasks, or SPTs). An SPT is presented by having the subject perform a minitask such as clapping his hands or opening a book in response to a verbal instruction. After a series of such tasks, the subject is given a free-recall test. A somewhat similar procedure was used by Saltz and DonnenwerthNolan (1981), who required subjects to encode sentences by pretending to enact their content. It should be noted, however, that whereas Saltz and DonnenwerthNolan used the enactment procedure to enhance the cued recall of the words that constituted the sentences, the emphasis in our studies has been on the free recall of the tasks, rather than on the verbal instructions. In fact, the verbal instructions in SPT memory are regarded primarily as a means of inducing the subject to generate the to-be-recalled SPT events (see Cohen, 1981).

In comparing performance on SPT lists and word lists, it was found that several of the properties associated with word recall did not generalize to SPT recall. More specifically, word memory exhibits strategic properties, but SPT memory does not. The evidence for this comes from several sources. First, subjects invariably report that they use strategies during word list presentation, but not during SPT presentation (Cohen, 1981). These subjective reports are partially supported by the absence of primacy in SPT recall but the presence of a well-

This research was supported by Grant A7023 from the Natural Sciences and Engineering Research Council of Canada. The author is grateful to J. M. Lacroix for his comments on an earlier draft of this paper, and to A. M. Glenberg, D. G. Elmes, H. L. Roediger III, and W. B. Whitten for their helpful critiques. Reprints may be ordered from R. L. Cohen, Department of Psychology, Glendon College, 2275 Bayview Avenue, Toronto, Ontario M4N 3M6, Canada. defined primacy effect in word recall-primacy presumably reflecting the use of cumulative rehearsal or other grouping strategies (see, e.g., Atkinson \& Shiffrin, 1971, and Rundus \& Atkinson, 1970). Second, the usually reliable levels-of-processing effect found in word-recall studies (Craik \& Tulving, 1975; Hyde \& Jenkins, 1969) has not been obtained in SPT recall (Cohen, 1981). This result suggests that SPTs may be processed at a uniform level regardless of which features of the events are attended to. Such a uniformity in encoding would also be consonant with a nonstrategic view of SPT recall. Third, Cohen and Stewart (1982) reported that, although a significant developmental effect was obtained in the free recall of word lists, age had no significant effect on SPT recall, at least from the age of 9 years and upward. This latter property also supports the notion that memory for SPTs is nonstrategic when considered in the context of Brown's (1975) argument that strategic tasks should show developmental differences. A similar result was obtained in a study that compared educable mentally retarded (EMR) subjects with nonretarded controls (Cohen \& Bean, 1983). The EMR subjects showed a significant deficit in immediate word, but not immediate SPT, recall. Since retarded individuals are presumed to have a general strategic deficit and should therefore be penalized in any strategic memory test (Campione \& Brown, 1978), this result is also indicative of the nonstrategic nature of SPT recall.

It should be noted that it is not simply the type of stimulus materials, words, or action events that is responsible for these differences in properties. If, instead of performing the tasks him- or herself, the subject views the experimenter performing them (experimenterperformed tasks, or EPTs), subsequent recall does show a significant primacy effect, recall is reliably affected when attention is directed to "deep" versus "shallow" features of the tasks (Cohen, 1981), and a significant 
EMR-controls difference is obtained (Cohen \& Bean, 1983). Similar results have been observed using the verbal instructions as list events, without either the subject's or the experimenter's translating them into actions (Cohen, 1981; Cohen \& Bean, 1983). In light of these data, the failure of the SPTs to conform to the laws governing word memory has been ascribed to the SPT procedure's involving the subjects in enacting the events.

In the case of word events, then, our previous results are entirely consistent with the current view that such variables as age, IQ, and levels-of-processing instructions affect the manner in which these events are encoded, which in turn affects recall levels. That such results have not been obtained with SPTs suggests that the encoding of these events is not affected by age, IQ, or levels-ofprocessing instructions, or if it is, that the resulting differences are trivial in determining recall performance. The present investigation aimed to further explore possible differences in the properties of word and SPT recall, with special regard to the relationship between encoding variables and recall level.

As already stated, our previous attempt to influence the recall of SPTs through the use of the levels-ofprocessing procedure was unsuccessful (Cohen, 1981). In that study, the object of the acquisition manipulation was to direct the subject's attention to different aspects of the events. The first experiment of our present research again attempted to vary recall level by manipulating the subject's attention during the acquisition phase of a free-recall task. In this task, the intention was to focus more of the subject's attention on certain items than on others, by designating them as being especially im. portant to remember.

Earlier studies have demonstrated the effectiveness of similar procedures in determining the recall levels of verbal events. For example, designating each pair as being important to remember or not so important to remember simultaneously with its presentation affected recall performance in the expected direction, in a pairedassociate task (Harley, 1965a, 1965b). Similarly, in freerecall tasks, an instruction to forget certain words, given prior to or subsequent to their presentation, has been shown to reduce the probability of their recall, as compared with the probability of recall of items not accompanied by a forget instruction (Bjork, 1972; Bjork \& Woodward, 1973). Given the aforementioned findings, it was expected that the free recall of word events in Experiment 1 would be influenced by the importance instruction, with more favored than unfavored words being recalled.

In contrast, the previously demonstrated nonstrategic properties of SPT events, and especially their resistance to the levels-of-processing manipulation, suggested that SPT recall might not be affected by differentially weighting the importance of the events.

Up to this point, our interest has been concentrated on possible dependencies between encoding variables and recall level within items. In other words, we have studied how age, IQ, and processing instructions affect the recall of a given event (actually, a series of given events). Experiment 2 aimed to extend our earlier work by looking at possible relationships between encoding variables and level of recall between items. This was accomplished by having subjects rate, during acquisition, how likely they were to recall each event, and then by testing the accuracy of the ratings against subsequent event recall. It has already been demonstrated that, in the case of verbal events, subjects are able to categorize items according to how easily the items will be memorized (see Zechmeister \& Nyberg, 1982). In lists of verbal events, then, items apparently vary in the impact that they make on subjects during encoding, and this variation in encoding impact is predictive of how readily the items will be recalled. Similar results were expected in the case of the word events in Experiment 2.

For SPT recall, the outcome was not so readily predictable. Even if our previous SPT data stemmed from a failure of our procedures to affect the manner of SPT encoding, it could still be possible for encoding impact to vary across the events in our SPT pool. The main interest in Experiment 2, then, was in whether or not subjects showed patterns of SPT recallability ratings similar to the patterns obtained from the word events, and, if so, whether these ratings would be predictive of actual recall performance.

\section{EXPERIMENT 1}

Experiment 1 used a free-recall procedure in order to measure the effect of varying the importance assigned to list events on subsequent recall. This was done by designating half of the items in each list as being especially important to remember.

The conditions of main interest used lists of words or SPTs. In our earlier studies, word events were presented auditorily. Auditory word lists differ from SPT lists not only in that SPT events have substantial nonverbal components, but also in that SPT items have visual components. To control for this possible confounding of presentation modality, two word conditions were included in the study, one using auditory presentation and the other, visual presentation.

If differences were to be found between word and SPT performance, it would obviously be of interest to specify which feature of the two types of material was responsible for this difference. For this reason, two further conditions were included in this study. One of these used the EPTs referred to earlier. This condition resembled the SPT condition in its use of nonverbal, everyday action events. It resembled the word condition, however, in the sense that the subjects were free to attend or not attend to the items during presentation. And finally, a comparsion of the word events and the action events reveals an additional difference, namely, that each word event consists (obviously) of one word, whereas each EPT or SPT has a potential verbal component (the 
instruction), which consists of a sentence. To control for the possible influence of this difference, a fifth condition, in which the events consisted of the task instructions used in the EPT and SPT conditions, without the tasks being translated into overt action, was included.

Since previous data had suggested that both EPT and instruction memory can be affected by encoding manipulations (see the introduction), it was expected that the recall of these events would be influenced by their designated importance.

\section{Method}

Materials. Forty-two two-syllable words having a frequency of 20 or more per million (Kučera \& Francis, 1967) were selected from the Toronto Word Pool. These were assigned randomly to two lists, each of 21 words. The assignment procedure was repeated thrice more, giving four different pairs of lists. These lists were used for both the auditory- and visual-word conditions.

Forty-two tasks were selected from Cohen's (1981) task pool and were used to contruct four pairs of task lists, using the same procedure as with the word lists. These lists were used for the SPT, EPT, and instructions conditions.

In all lists, the three primacy and four recency events were considered to be buffer items. The experimental materials proper consisted of the 14 nonbuffer items from the middle of each list.

Subjects. Eighty weekend visitors to the Ontario Science Centre in Toronto volunteered to take part in this experiment. These were in the age range 18-50 years. They were assigned randomly to the five conditions, 16 to each condition, 4 subjects to each of the four list pairs within each condition. Testing was on an individual basis.

Procedure. Each subject was informed that a memory test consisting of two lists of events would be administered. The particular class of event (EPT, SPT, etc.) to be included in the testing session was described, and the subject was told that, following each list, he was to recall as many items as possible, in any order. The subject was instructed further that it was more important to recall some of the items in the lists than others. A favored item would be indicated by the experimenter's pointing to the subject just prior to its presentation.

A randomly chosen 7 of the 14 midlist items in each list were designated as being important to remember. Counterbalancing was achieved within each subset of lists by designating those items that were favored for two of the subjects as being nonfavored for two other subjects and, of course, vice versa.

For the SPT lists, the subject sat at a table, half of which was screened from view. Some of the tasks involved the use of objects, whereas others did not. The objects required for task performance were concealed from the subject behind the screen. The experimenter presented each task by reading aloud a verbal instruction (e.g., "Knock on the table"), following which the subject enacted the task. When the task required the use of an object, the object was presented together with the instruction for example, the experimenter would present a toothpick with the instruction "Break the toothpick." Immediately following the task's completion, the experimenter retrieved the object and replaced it behind the screen. Following each list, the experimenter pointed to a note pad in front of the subject, this being the signal for the subject to write down as many of the tasks as he or she could recall, in any order.

The EPT lists were presented in a similar manner, with the experimenter's reading aloud a series of task instructions. Instead of the tasks' being enacted by the subject, however, they were enacted by the experimenter.

In the instructions and auditory-word conditions, the items were read aloud by the experimenter. In the visual-word con- dition, the items were presented on flash cards, each card being exposed for approximately $4 \mathrm{sec}$.

The rate of presentation for words, instructions, and EPTs was completely under the control of the experimenter and was set at 1 event $/ 6 \mathrm{sec}$. SPT presentation, which was partly under the control of the subject, had a somewhat slower rate, approximately 1 event $/ 8 \mathrm{sec}$.

The recall responses in the word conditions consisted of single words. In the other three conditions, the subjects were instructed to describe each recalled event by a phrase of two or three words. Since it takes less time to actually write one-word responses than responses comprising several words, subjects in the two word conditions were allowed $90 \mathrm{sec}$ to recall each list, whereas subjects in the other three conditions were allowed $2 \mathrm{~min}$.

Data analysis. Unlike for word recall, the scoring of recall responses made in the SPT, EPT, and instructions conditions requires some comment. The subjects' responses in these three conditions were almost exclusively in accordance with the experimenter's directive to describe each recalled event by a phrase of two or three words. In the case of SPTs, EPTs, and instructions, a response was scored as correct if it enabled the unequivocal identification of an event in the appropriate list. In fact, the event descriptions used in recall were generally similar to those used by the experimenter during presentation. Probably the most distorted description obtained in the study was the recall of the task "name the street you live on" as "remember your street"' (scored as correct).

\section{Results and Discussion}

Neither the level of recall nor the effect of the importance variable showed a systematic variation over the two lists in any of the five conditions. The data in each condition were therefore collapsed over lists. The results are given in Table 1. Although recall shows some effect of the importance variable in all five conditions, the ratios given in row 3 of Table 1 suggest an interaction between the class of event and the importance variables. The verbal events, and expecially the auditory words, show a relatively large effect of the importance manipulation, whereas the two types of action events show a very modest effect. An analysis of variance yielded a significant $(\mathrm{p}<.05)$ class of event $\mathrm{x}$ importance interaction $[\mathrm{F}(4,75)=2.51, \mathrm{MSe}=3.31]$, although posttesting showed designated importance to have a significant effect in all five conditions.

The validity of the ratios given in row 3 in Table 1 as indicators of the effect of the importance variable is supported by omega ${ }^{2}$ analyses. Although importance was a within-subjects variable, Tukey's test showed that

Table 1

Proportion of High- (HI) and Low-Importance (LI) Events Recalled Under the Five Conditions of Experiment 1

\begin{tabular}{|c|c|c|c|c|c|}
\hline & $\begin{array}{l}\text { Auditory } \\
\text { Words }\end{array}$ & $\begin{array}{l}\text { Visual } \\
\text { Words }\end{array}$ & Instructions & EPTs & SPTs \\
\hline $\mathrm{HI}$ & .46 & .48 & .37 & .50 & .52 \\
\hline LI & .23 & .30 & .26 & .44 & .44 \\
\hline Ratio HI/LI & 2.00 & 1.60 & 1.42 & 1.14 & 1.18 \\
\hline $\mathrm{Omega}^{2}$ & .55 & .12 & $.14 *$ & .04 & .06 \\
\hline
\end{tabular}

*Note that this value is an underestimate. 
the additive model was appropriate for analyzing the data from the two word conditions, the EPT condition, and the SPT condition. Thus, it was possible to calculate accurate omega ${ }^{2}$ values for the importance variable in these latter four conditions (Myers, 1979). The data from the instructions condition did not, however, fit an additive model. In this case, the omega ${ }^{2}$ value is an underestimate of the proportion of the variance associated with the importance variable (see Vaughan \& Corballis, 1969).

The omega ${ }^{2}$ values for the five important/unimportant comparisons, given in the last row of Table 1, tell us that the importance variable produced a considerable effect in the auditory-words condition, but not in the two action-events conditions. In the case of the visual words, the failure of the omega ${ }^{2}$ value to correspond with the ratio value given in row 3 of Table 1 depends on the high error variance associated with this condition. In the action-events conditions, however, the low omega ${ }^{2}$ values appear to be true indicators of small effects.

Although the effects of designated importance, as measured by the omega ${ }^{2}$ values, are not precisely as predicted, the main trend of the data is in general agreement with our expectations. The auditory words showed a substantial effect of the differential importance weightings; in the case of the SPTs, this effect, although significant, was minimal.

Two of the conditions in Experiment 1 did, however, yield somewhat surprising data. First, in the light of the large omega ${ }^{2}$ value obtained for auditory words, the rather meager omega ${ }^{2}$ value found in the visual-word condition was quite unexpected. Given the strong relationship between recall level and attention-directing instructions found in other studies using visual words (Bjork \& Woodward, 1973; Craik \& Tulving, 1975), it is probably safer to regard the difference between the auditory- and visual-word data in Experiment 1 as an experimental anomaly rather than an effect of presentation modality.

And second, the fact that EPT recall showed the same minimal sensitivity to the acquisition variable as did SPT recall deserves some comment. This will be deferred, however, until the final discussion.

\section{EXPERIMENT 2}

Our second study explored further relationships between recall level and encoding variables. Specifically, interest was centered on how accurately subjects would be able to predict, at acquisition, the recallabilities of individual events. To this end, the subjects were presented with lists of events in a free-recall test. Following each event, the subjects were to rate whether or not they thought they would recall it on the subsequent memory test. The relationship between recall level and encoding impact could then be measured by conditionalizing recall level on the predictions.

\section{Method}

Materials. This experiment used the same materials as did Experiment 1 . The 42 items in each pool were split into pairs of 21 item lists three times, to give three versions of the list pairs, instead of the four versions used in Experiment 1.

The word lists were presented in the visual as well as in the auditory modality; the task lists were presented in the form of SPTs, EPTs, and instructions.

Subjects. The subjects again were weekend visitors to the Ontario Science Centre. Seventy-five volunteers, in the age range 18-50 years, were assigned randomly to the five conditions of the experiment, 15 to each condition, 5 subjects to each list pair. The subjects were tested individually.

Procedure. Each subject was informed of the aim of the study, namely, that we were testing how well people could predict which items they would recall. The makeup of the appropri ate event lists was described, and free recall instructions were given, as in Experiment 1. The subject was then supplied with a sheet with two columns of 21 numbered spaces in which the ratings were to be written. The events were to be rated on a 4-point scale, which ranged from +2 (fairly sure the event would be recalled) to -2 (fairly sure that the item would not be recalled), with no neutral zero point. It was explained that each event was to be rated immediately following its presentation.

In each of the five conditions, event presentation was as in Experiment 1. Since the subjects' ratings were self-paced, the rates of presentation were partially under the subjects' control in all conditions. The approximate presentation rate was 1 event/ $6 \mathrm{sec}$, except in the case of the SPTs, which required approximately $8 \mathrm{sec}$, on the average, to present each event. Ninety seconds were allowed for the written recall of each word list, and $2 \mathrm{~min}$ were allowed for the recall of task and instructions lists.

\section{Results}

As in Experiment 1, the three primacy and four recency items were considered to be buffers, thus leaving the 14 midlist items to provide the relevant data. Also as in Experiment 1, the data were collapsed over lists.

In each of the five conditions, 2 subjects predicted (unrealistically) that they would recall 12 or more of the 14 critical items. In addition, 1 subject in the visualword condition predicted that he would not recall 12 of the 14 items in one of the lists. Since the proportions of recalled/recall-predicted events and recalled/recall-notpredicted events calculated for these 11 subjects would have been rather meaningless, their data were discarded. The numbers of subjects were equated for the five conditions, however, by testing an additional subject in the visual-word condition.

The subjects' rating behavior is shown in Table 2. Although there are some differences in the distributions of

Table 2

Distributions of Recall Predictions Across Rating Categories, for the Five Conditions of Experiment 2

\begin{tabular}{lrrrr}
\hline & \multicolumn{4}{c}{ Rating } \\
\cline { 2 - 5 } \multicolumn{1}{c}{ Condition } & +2 & +1 & -1 & -2 \\
\hline Auditory Words & 67 & 115 & 113 & 69 \\
Visual Words & 50 & 144 & 139 & 31 \\
Instructions & 51 & 158 & 95 & 60 \\
EPTs & 53 & 157 & 126 & 28 \\
SPTs & 113 & 103 & 94 & 54 \\
\hline
\end{tabular}


the judgments among the rating categories, across the five subject groups, these are overshadowed by the obvious similarities in the behavior of the subjects in the five conditions. The distribution of the ratings indicates that the events varied widely in their subjective encoding impact, not only in the verbal-events conditions, but also in the EPT and SPT conditions.

Figure 1 shows the proportion of items recalled from each of the four prediction categories. Visual inspection of these curves suggests that the five classes of memory events represent a continuum ranging from a strong relationship between recall predictability and recall level in the case of word events to a negligible relationship in the case of the SPTs. In the three verbal conditions (top three graphs), the proportion of events recalled shows an unmistakable decrease as predicted recall confidence decreases. The fourth graph shows some arguable tendency for the proportion of EPTs recalled to be related to predictive confidence. In the bottom graph, the recall of the SPTs appears to be completely independent of predictive confidence rating.

Since some subjects did not use one or both of the two extreme rating categories, the prediction ratings were collapsed into two categories, namely, recall predicted and recall not predicted, for the purpose of statistical analysis. The predictive capabilities of the subjects under the five conditions were then measured by com-

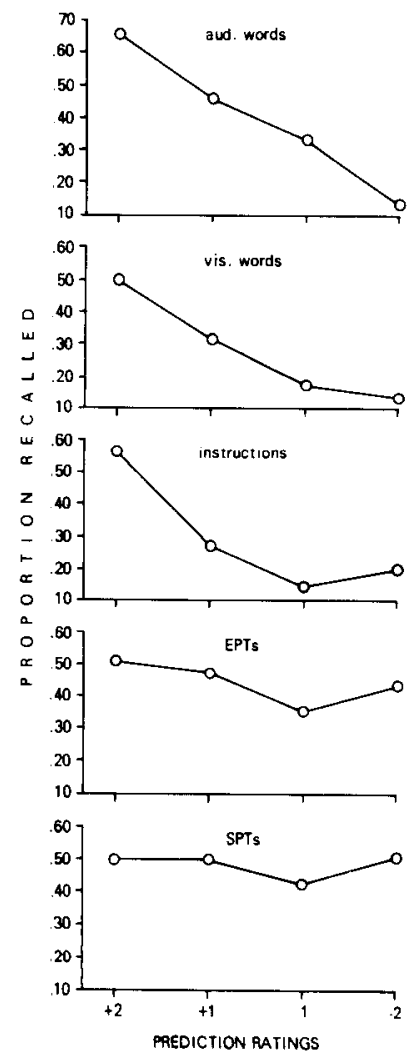

Figure 1. Proportion of items recalled as a function of prediction rating, for the five classes of event.
Table 3

Proportion of Events Recalled From Each of the Two Prediction Categories, Recall Predicted (RP) and Recall Not Predicted (RNP), in Experiment 2

\begin{tabular}{|c|c|c|c|c|c|}
\hline & $\begin{array}{l}\text { Auditory } \\
\text { Words }\end{array}$ & $\begin{array}{l}\text { Visual } \\
\text { Words }\end{array}$ & Instructions & EPTs & SPTs \\
\hline RP & .54 & .40 & .34 & .50 & .50 \\
\hline RNP & .27 & .19 & .18 & .38 & .47 \\
\hline Ratio RP/RNP & 2.00 & 2.11 & 1.89 & 1.32 & 1.06 \\
\hline Omega $^{2}$ & .57 & .63 & .55 & .18 & .00 \\
\hline
\end{tabular}

paring the proportions of recall-predicted and recall-notpredicted items actually recalled. These data, expressed as ratios in row 3 of Table 3, reiterate the observations apparent in Figure 1, namely, that the verbal conditions provided an opportunity for predicting recall with some degree of accuracy, whereas the SPT condition did not. The EPT condition also produced a recall advantage for recall-predicted items, although this was more modest than those obtained in the verbal conditions.

Analyses of variance were performed on the Table 3 data, once again using the untransformed proportions and once using arcsine transformations of these proportions. Both analyses yielded a significant $(p<.05)$ interaction of event class with the prediction variable $[F(4,60)=$ 2.81 , MSe $=.02$ (untransformed data) and $\mathrm{F}(4,60)=$ $2.89, \mathrm{MSe}=.03$ (transformed data) $]$.

Omega $^{2}$ values for the prediction variable in each of the five conditions are given in the last row of Table 3. Since Tukey's test indicated that the additive model could be applied in all conditions, all five omega ${ }^{2}$ values should be accurate indicators of the relationship between rated recallability and recall level. In each of the three verbal conditions, the prediction variable accounts for more than $50 \%$ of the variance in the data. In the EPT condition, only $18 \%$ of the variance is accounted for by the prediction variable. This value is, however, sufficiently greater than the zero variance associated with the prediction variable in the SPT condition to suggest at least a quantitative difference between these two classes of action events, with respect to a connection between encoding impact and subsequent recall.

Finally, the serial position data from Experiments 1 and 2 were examined for possible differences between the conditions. These are shown in Figure 2. As in Figure 1, the curves in Figure 2 may be ranked in a continuum from the word to the action event conditions. Scanning Figure 2 from top to bottom, it is observed that the curves progressively lose the $U$ shape traditionally associated with free recall. In particular, the clearly defined primacy effects apparent in the word conditions are lost.

Insofar as primacy is a valid indicator of a cumulative strategy, the curves in Figure 2 suggest that such a strategy was used in the word but not in the SPT conditions. The equivalence of the SPT and EPT conditions in this respect appear to depend on the rather unusual acquisition conditions used in Experiments 1 and 2. As already 


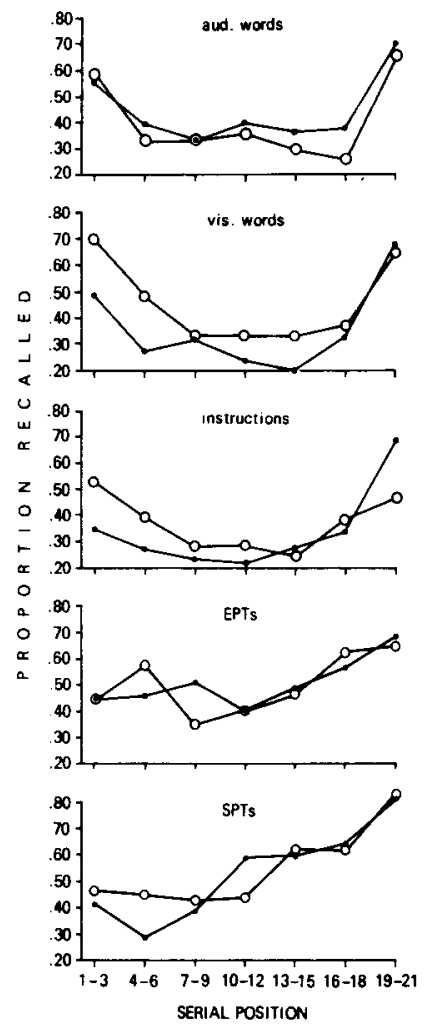

Figure 2. Recall plotted as a function of serial position for all five classes of event. Experiment 1,

$\leadsto$, Experiment 2,

mentioned, EPT recall, but not SPT recall, shows a clear primacy effect under more traditional free-recall conditions.

The recency effects also differentiate between the words and action events. The word lists show the steep, narrow-span recency effects typical of standard free recall; the EPT and SPT lists show a more extensive recency of more gradual slope. Such gently sloping recency effects can also be obtained with lists of verbal events, using what Poltrock and MacLeod (1977) called the continuous distractor paradigm. In this procedure, each item is isolated during presentation by having the subject perform some mental task during the interitem in terval (see Bjork \& Whitten, 1974). The isolation of the items is further accentuated by instructing subjects to limit their rehearsal to the currently presented event. The gradual recency effects in the task-recall conditions, being similar to the effects obtained under continuous distractor conditions, may also be taken as an indication that each action event was encoded separately from its neighbors.

\section{DISCUSSION}

The distinction between word events and SPTs, made in the introduction, receives further support from our present studies. Reiterating the main points of evidence to date yields the following picture. First, subjective reports suggest that acquisition strategies are used spontaneously in encoding words but not SPTs. Second, our prior studies have demonstrated a significant recall deficit in children and EMR individuals in the immediate free recall of word, but not SPT, lists. Third, the imposition of encoding strategies through the use of the levelsof-processing manipulation or by differentially weighting the importance of list events produces dramatic effects in word recall but little or no effect in SPT recall. And fourth, encoding impact is predictive of later recall of words but not of SPTs.

These data are clearly consistent with the strategic view of word memory. Experimental manipulations such as variations in level of processing or designated importance affect the manner in which words are encoded, which in turn affects the likelihood of their recall. Similarly, samples from different populations (adults, children, EMRs) presumably vary in strategic efficiency, which is again reflected in the recall levels.

By the same token, the SPT data are consistent with the notion that memory for these events is largely nonstrategic. There are three levels of argument for this conclusion. First, attempts to manipulate encoding through the use of attention-directing procedures have yielded, at best, a weak effect on SPT recall. A basic principle in event memory states that the likelihood of remembering an event depends on how well the event is encoded (Tulving, 1983). Since there is no reason at this point for supposing that SPT recall constitutes an exception to this principle, it is presently concluded that the relative failure of our attempts to influence recall level by means of encoding manipulations reflects an inflexibility in the way SPTs are encoded, rather than a breakdown of the encoding-recall principle. This conclusion is, of course, somewhat tentative, since future research may well uncover an encoding manipulation that does exert a substantial influence on SPT recall.

The second level of the argument revolves around the subjective reports. Classifying a recall task as being strategic carries the implication that subjects consciously attempt to steer the encoding of the to-be-remembered material so as to optimize subsequent recall performance. Those same subjects who claimed that they had spontaneously used such encoding strategies in word recall reported that, in SPT recall, they simply performed each task as it was presented, without making any effort to memorize it (Cohen, 1981). Assuming that the class of event, words or SPTs, does not affect motivation, it would appear that, when faced with the SPT test, subjects judge that any attempt to use mnemonic strategies would not pay off in subsequent performance. In other words, subjects behave as if they had knowledge of the results of the encoding-manipulation studies.

The third level of the argument involves consideration of our earlier individual-differences data (Cohen \& Bean, 1983; Cohen \& Stewart, 1982). Since both young children and EMR individuals should show deficits in stra- 
tegic memory tests, the absence of such deficits in SPT recall again points to the nonstrategic nature of SPT memory.

One result that is somewhat equivocal is that obtained in Experiment 2. On the one hand, the dissociation between the encoding impact of SPTs and their subsequent recall fits the general picture of a minimal relationship between encoding variables and recall level. On the other hand, the ratings made during the acquisition phase of Experiment 2 suggest that subjects judge SPTs to possess some attribute (or attributes), to varying degrees, that determines the likelihood of subsequent recall. In other words, subjects appear to believe that SPT recall may be influenced by some encoding variable that is intrinsic to the SPTs, but that they themselves cannot influence. That the predictions were not borne out by the recall performances raises the question of whether or not the SPTs do in fact possess an encoding attribute that determines recall but that our subjects failed to detect. The present data do not allow us to answer this question, and it is presently under further investigation.

The discussion so far has emphasized the contrast between word and SPT events, without making any mention of instruction and EPT recall. Whereas instructions may be readily categorized with words as strategic memory events, EPT events are somewhat more difficult to classify, since they sometimes behave like SPTs and sometimes like words. In fact, the results of the above two experiments suggest that the words and SPTs may represent two anchor positions on a continuum of memory events, with the instructions and EPTs occupying intermediate positions.

In Experiment 2, the relationships between encoding impact and level of recall appear to form a continuum from virtually a zero relationship in the case of SPTs to a well-defined relationship in the case of the words (see Figure 1). A similar ranking of the memory events is apparent when considering the serial position effects in Figure 2. In Experiment 1, this trend is again apparent, especially in the ratios of recalled important to recalled unimportant events (see Table 1). Thus, the data suggest a continuum ranging from words, through instructions, to EPTs and SPTs, whose dimensions may be strategy use (as suggested by the serial position data) and/or automaticity in encoding (as suggested by the results of both experiments).

A parallel can be drawn between the continuum that has emerged here and two other continua, one in the animal learning area and one in memory. In proposing a preparedness-of-associations continuum, Seligman (1970) pointed out that, although associations between various stimuli and responses can range from readily learnable (prepared associations) to virtually unlearnable (contraprepared associations), traditional learning research has been concentrated almost exclusively in the middle of this range (unprepared associations). In the memory area, Hasher and Zacks (1979) proposed a similar continuum, from effortful encoding (attention de- manding) to automatic encoding (requires no attention). The point is made that traditional memory research has been narrow in its scope, by concentrating on the study of memory tasks that involve effortful encoding. The continuum proposed here is somewhat similar to that of Hasher and Zacks, since the use of strategies is effortful. The differences between the continua are, however, twofold. First, Hasher and Zacks's continuum appears to range from event encoding at the effortful end to the encoding of ancillary information such as frequency of occurrence, spatial information, and temporal information at the automatic end. The continuum that is proposed here only has reference to, and is anchored at both ends by, memory for events. And second, although strategic processes are effortful, it is not necessarily true that the encoding of events such as SPTs does not demand attention, even though such encoding appears to be nonstrategic.

Regardless of any differences in these continua, however, the argument may be reiterated that event memory has been studied almost exclusively in the word region. It is perhaps time that memory for events from the other end of the continuum receives some attention. If nothing else, research into the recall of action events might facilitate the study of retrieval processes uncontaminated by variations in encoding.

\section{REFERENCES}

Atrinson, R. C., \& Shiffrin, R. M. The control of short-term memory. Scientific American, 1971, 225(2), 82-90.

BJoRk, R. A. Theoretical implications of directed forgetting. In A. W. Melton \& E. Martin (Eds.), Coding processes in human memory. Washington, D.C: Winston, 1972.

Bjork, R. A., \& Whitten, W. B. Recency-sensitive retrieval processes in long-term free recall. Cognitive Psychology, 1974, 6, 173-189.

Bjork, R. A., \& Woodward, A. E. Directed forgetting of individual words in free recall. Journal of Experimental Psy. chology, 1973, 99, 22-27.

Brown, A. L. The development of memory: Knowing, knowing about knowing, and knowing how to know. In $\mathrm{H}$. W. Reese (Ed.), Advances in child development and behavior (Vol. 10). New York: Academic Press, 1975.

Campione, J. C., \& Brown, A. L. Toward a theory of intelligence: Contributions from research with retarded children. Intelligence, 1978, 2, 279-304.

Conen, R. L. On the generality of some memory laws. Scandinavian Journal of Psychology, 1981, 22, 267-281.

Cohen, R. L., \& BEAN, G. Memory in educable mentally retarded adults: Deficit in subject or experimenter? Intelligence, 1983, 7, 287-298.

Cohen, R. L., \& Stewart, M. How to avoid developmental effects in free recall. Scandinavian Journal of Psychology, 1982, 23, 9-16.

Craik, F. I. M., \& Tulving, E. Depth of processing and the retention of words in episodic memory. Journal of Experimental Psychology: General, 1975, 104, 268-294.

HARLEY, W. The effect of monetary incentive in paired associate learning using a differential method. Psychonomic Science, 1965, 2, 377-378. (a)

HARLEY, $W$. The effect of monetary incentive in paired associate learning using an absolute method. Psychonomic Science, 1965, 3, 141-142. (b)

Hasher, L., \& Zacks, R. T. Automatic and effortful processes 
in memory. Journal of Experimental Psychology: General, $1979,108,356-388$.

Hyde, T. S., \& Jenkins, J. J. Differential effects of incidental tasks on the organization of recall of a list of highly associated words. Journal of Experimental Psychology, 1969, 82, 472-481.

Kučera, H., \& Francis, W. N. Computational analysis of present-day American English. Providence: Brown University Press, 1967.

Myers, J. L. Fundamentals of experimental design (3rd ed.). Boston: Allyn \& Bacon, 1979.

Poltrock, S. E., \& MacLeod, C. M. Primacy and recency in the continuous distractor paradigm. Journal of Experimental Psychology: Human Learning and Memory, 1977, 3, 560-571.

Rundus, D., \& Atkinson, R. C. Rehearsal processes in free recall: A procedure for direct observation. Journal of Verbal Learning and Verbal Behavior, 1970, 9, 99-105.

Saltz, E., \& Donnenwerth-Nolan, S. Does motoric imagery facilitate memory for sentences? A selective interference test. Journal of Verbal Learning and Verbal Behavior, 1981, 20, 322-332.

Seligman, M. E. P. On the generality of the laws of learning. Psychological Review, 1970, 77, 406-418.

Tulvina, E. Elements of episodic memory. Oxford: Clarendon Press, 1983.

Vaughan, G. M., \& Corballis, M. C. Beyond tests of significance: Estimating strength of effects in selected ANOVA designs. Psychological Bulletin, 1969, 72, 204-213.

Zechmeister, E. B., \& Nyberg, S. N. Human memory: An introduction to research and theory. Monterey, Calif: Brooks/ Cole, 1982.

(Manuscript received March 14, 1983;

revision accepted for publication August 10, 1983.) 